\title{
Análise dos efeitos das incertezas nas estimativas de tempos de falha de produtos via ensaios acelerados
}

\author{
Maria Célia de Oliveira Papa \\ Alvaro J. Abackerli \\ Paulo Augusto Cauchick Miguel \\ UNIMEP; FEAU
}

\begin{abstract}
Resumo
As empresas do setor industrial têm hoje o desafio de lançar rapidamente produtos mantendo, entretanto, sua confiabilidade em níveis progressivamente crescentes. 0 método mais usual para reunir informações sobre a confiabilidade de novos produtos é o ensaio acelerado (ALT), de onde o fabricante reúne informações sobre a vida e sobre os prazos de garantia. Um ALT requer a submissão do produto a condições de estresse para coletar seus tempos de falha, e para isso é necessário um experimento que materialize cargas de estresse nominalmente definidas. Entretanto, cargas de estresse experimentais estão sempre sujeitas a incertezas que influenciam os resultados do ensaio e a informação sobre a confiabilidade. Aqui se investiga a influência das incertezas experimentais sobre estimativas de vida de relés eletromagnéticos, obtidas usando ATLs. Os resultados mostram que, sem uma análise adequada dos dados, os efeitos das incertezas podem ser relevantes mesmo quando o teste é executado em condições adequadas de chão-de-fábrica, o que conseqüentemente influencia as estimativas de tempo de vida dos produtos.
\end{abstract}

Palavras-chave

Desenvolvimento do produto, ensaio acelerado, incerteza, SIMEX.

\section{Uncertainty effect analysis on time to failure estimates through accelerated life tests}

\begin{abstract}
Industrial firms face today the challenge to launch new products in shorter times keeping however their reliability in increasing higher levels. The most used method for gathering reliability information for new products is the accelerated life test (ALT), from which the manufacturer can derive information about the product's life and then its warranty period. ALT requires submitting the product to stress conditions and acquiring its time to failure, for which it is necessary to set up experiments where the stress loads are physically and nominally defined. However, experimental stress loads are always subjected to uncertainties that influence the tests results and so the product's reliability. This paper investigates the influences of experimental uncertainties in life estimates of electromagnetic relays, obtained through ALTs. Results show that, without the proper data analysis the uncertainty effects on life estimates may be significant also when the tests are performed in adequate shop floor conditions that consequently influence the product's lifetime estimates.
\end{abstract}

Key words

Product development, accelerated life test, uncertainty, SIMEX. 


\section{INTRODUÇÃO}

Um dos desafios atuais das organizações é desenvolver novos produtos em tempo reduzido, atendendo aos requisitos de qualidade e confiabilidade (THEIJE et al., 1998). Para isso, diversos métodos e técnicas com essa finalidade são utilizados ao longo do processo de desenvolvimento de novos produtos.

Independentemente das estratégias adotadas para o desenvolvimento de novos produtos, a grande maioria delas inclui estudos de confiabilidade para assegurar que as especificações relacionadas à qualidade do produto sejam atendidas. A literatura (AHMED, 1996; ELLEKLAER; BISGARD, 1998; THEIJE et al., 1998; BOOKER, 2003) sugere vários destes estudos com aplicações que utilizam diferentes testes de confiabilidade.

A forma mais usual destes testes é o ensaio acelerado (ALT) (AW, 2005), pois permite obter informações de forma muito mais rápida do que seria possível se o produto fosse deixado falhar nas condições normais de uso. Um dos principais objetivos de um ensaio acelerado é obter rapidamente as estimativas de características da distribuição do tempo até a falha, tais como percentis, em particular a mediana, o tempo médio até a falha ou o percentual de falhas no tempo $t_{0}$, que é a missão estabelecida para o produto. Para que a aceleração da falha ocorra, o produto é colocado em funcionamento sob cargas de estresse que excedem as condições normais de uso. Uma das grandes vantagens de realizar estes testes de forma acelerada é a redução de tempo de lançamento do produto que trás uma vantagem competitiva, pois possibilita oportunidades mercadológicas (KAHN et al., 2006).

Os tempos de falha acelerada são extrapolados para as condições normais de uso por meio de regressões que utilizam modelos do tipo locação e escala. Os parâmetros destes modelos estão relacionados às condições do teste e aos mecanismos de falha do produto ensaiado, dados pelo parâmetro de locação, representado por uma relação estresse-resposta e pelo parâmetro de escala, oferecido por uma distribuição de probabilidade que modela os tempos de falha acelerados (NELSON, 2004).

Tanto na realização do ensaio como na análise dos dados as cargas de estresse são consideradas medidas exatas, ou seja, livres de incertezas. Porém, na prática experimental, tais cargas são definidas experimentalmente e isso não permite a sua definição absoluta pela existência de incertezas de medição (INMETRO, 2003). Assim, como não é possível assumir um valor verdadeiro para esta variável de estresse, adota-se um valor verdadeiro convencional para a sua definição experimental.
Por definição, este valor verdadeiro convencional deve ser adequado o suficiente para representar a quantia de interesse (INMETRO, 2003).

Em função dessa prática, para que os resultados das estimativas de tempos de falha obtidos por meio de ensaios acelerados sejam aceitáveis, é preciso que as incertezas associadas a eles sejam consideradas na análise e seus efeitos avaliados. Nesse sentido, o objetivo do presente trabalho é avaliar os efeitos das incertezas nas variáveis de estresse em ensaios acelerados. Para o desenvolvimento deste estudo foi utilizado um conjunto de dados reais de um ensaio acelerado de relés, realizado por Sasseron (2005). A partir destes dados foram feitos dois tipos de análise: a primeira, aqui denominada análise convencional, não considera as incertezas na variável de estresse e a segunda, considera estas incertezas. Para ambas as análises são obtidas as estimativas dos percentis dos tempos de falhas, que permitem comparar os resultados obtidos em ambas para concluir a respeito do efeito das incertezas sobre as estimativas dos tempos de falha e a vida esperada para o produto em função deles.

\section{I dos desafios atuais das organizações é desenvolver novos produtos em tempo reduzido, atendendo aos requisitos de qualidade e confiabilidade.}

A abordagem de pesquisa aqui discutida pode ser caracterizada como teórico-experimental e está fundamentalmente centrada na busca por uma resposta a um problema real de engenharia. Assim, o caráter experimental limita-se ao uso de dados reais e as suas conseqüências, tanto no delineamento metodológico do problema quanto na sua solução. Já o caráter teórico, centra esforços na busca por uma solução matemático-estatística adequada que considere as condições de contorno do problema real de engenharia e responda ao objetivo do estudo, mostrando sua obediência a estas condições reais de contorno, bem como a validade da solução obtida.

A solução foi implementada no software R-2. 5.1 (R Development Core Team, 2007) e submetida a testes de desempenho desenvolvidos como parte da solução metodológica do problema, cujos detalhes podem ser encontrados em Papa (2007). Para fundamentar teoricamente o presente estudo, primeiramente é apresentada uma síntese dos principais conceitos relacionados ao problema da obtenção das estimativas de tempos de falha do produto, seguida pelo método de análise que considera as incertezas e, finalmente, pelos resultados e a respectiva discussão. 


\section{O ENSAIO ACELERADO}

Um ensaio acelerado consiste de utilizar uma variedade de métodos que intencionalmente diminuem a vida útil de um produto ou, de outro modo, aceleram a sua degradação (NELSON, 2004).

O principal interesse na realização deste ensaio é obter os "tempos de falha do produto" mais rapidamente e deles a estimativa da sua confiabilidade. O primeiro passo para a realização de um ensaio acelerado é o seu planejamento, que é feito por meio de um plano de teste. Esta abordagem objetiva estabelecer meios planejados e organizados para acelerar a falha do produto. Nele, as principais características e os elementos do ensaio acelerado são determinados. Tais características e elementos incluem os níveis de estresse, o tamanho e número de amostras, o modo de falha esperado, o tipo de censura, entre outros. Mais detalhes sobre planos de ensaio acelerado podem ser verificados em Nelson (2004), Mac Kane et al. (2005).

Os modelos de regressão para extrapolação dos dados acelerados para as condições normais de uso são denominados modelos de locação e escala. Estes modelos são construídos para o logaritmo do tempo de falha $T$, ou seja, $Y$ $=\log (T)$. A principal característica destes modelos é que os tempos de falha $Y=\log (T)$ têm distribuição com parâmetro de locação $\mu(x)$, que depende da variável de estresse $x$, e parâmetros de escala $\sigma>0$ constantes. A partir destas características, o modelo de regressão do tipo locação e escala assume a forma da expressão (1), onde $\varepsilon$ é o erro aleatório independente de $x$ e de $Y=\log (T)$.

$$
Y=\mu(x)+\sigma \varepsilon
$$

\section{Onde}

$Y=\log (T)$, sendo $T$ tempos de falha

$\mu(x)$ é o parâmetro de locação, sendo $x$ a variável de estresse

$\sigma$ é o parâmetro de escala, onde $\sigma>0$

$\varepsilon$ é o erro aleatório independente de $x$ e $Y=\log (T)$

Entre as diversas formas assumidas por este modelo de regressão, Papa (2007) utilizou o modelo Potência Inversa - log-normal para a extrapolação dos tempos de falha acelerados dos relés. Este modelo supõe que tais tempos de falha sejam ajustados pela distribuição log normal e a relação estresse-resposta é a de Potência Inversa.

Esta relação é utilizada para vários tipos de variável de estresse, exceto a temperatura. Por exemplo, lâmpadas incandescentes, isolantes, dielétricos, entre outros que envolvem variáveis como tensão e corrente (FREITAS; COLOSIMO, 1997). Supondo que a carga de estresse assuma valores positivos, a relação tem a forma dada pela expressão (2), onde
$\tau(V)$ é o tempo de falha, $A$ e $\omega$ são parâmetros do modelo, a serem estimados, e $V$ é a variável de estresse, como segue

$$
\tau(V)=\frac{A}{V^{\omega}}
$$

\section{Onde}

$\tau(V)$ é o tempo de falha

$A$ e $\omega$ são os parâmetros do modelo

$V$ é a variável de estresse, $\operatorname{com} V>0$

A forma linearizada da relação (equação 2) com parâmetros $\alpha=\ln (A), \beta=\omega$ é dada por

$$
\ln (\tau)=\alpha+\beta[-\ln (V)]
$$

A distribuição de probabilidade log-normal é estreitamente relacionada à distribuição normal. Como o nome sugere, o logaritmo de uma variável com distribuição lognormal de parâmetros $\mu$ e $\sigma$ tem uma distribuição normal com média $\mu$ e desvio padrão $\sigma$. Isso equivale a dizer que os dados provenientes de uma distribuição log-normal podem ser analisados segundo uma distribuição normal, desde que seja considerado o logaritmo da variável dependente (tempos de falha) no lugar de seus valores originais (COLOSIMO; GIOLO, 2006).

Assim sendo, assumir o modelo de regressão Potência Inversa - log-normal implica nas seguintes suposições:

- Para cada nível de estresse $V$, os tempos de falha do produto seguem uma distribuição log-normal. De forma equivalente, os logaritmos dos tempos de falha seguem uma distribuição Normal;

- O desvio padrão $\sigma$ da distribuição de $Y=\log (T)$ é constante, ou seja, independente da variável de estresse;

- O valor médio $\mu(x)$ da distribuição de $Y=\log (T)$ é uma função linear de $V$ da seguinte forma

$$
\log [\mu(V)]=\alpha+\beta V
$$

- A vida mediana da distribuição de $Y=\log (T) \tau_{50}$ é uma função linear de $V$ da seguinte forma:

$$
\log \left[\tau_{50}(V)\right]=\frac{10^{\alpha}}{V^{\beta}}
$$

Estas suposições produzem a função de distribuição acumulada do tempo de falha do produto $F(t ; V)$, dada pela equação (6), e seus respectivos percentis $t_{p}(V)$ equação (7) para um nível de estresse qualquer $V$.

$$
F(t ; V)=\Phi\left\{\left[\frac{\log (t)-\mu(-\log (V))}{\sigma}\right]\right\}
$$




$$
t_{p}(V)=\mu(x)+z_{p} \sigma
$$

O modelo de regressão de locação e escala é então utilizado para gerar regressões que permitem extrapolar os dados acelerados para as condições normais de uso. Para isso, é necessário estimar os parâmetros deste modelo. Em situações com dados que possuem tempos completos e censurados, o estimador utilizado é o de máxima verossimilhança (MONTENEGRO, 2006). Segundo Cordeiro (1992), a verossimilhança é interpretada como uma medida de crença racional para se chegar a conclusões baseadas nos dados. $\mathrm{O}$ método de máxima verossimilhança não contradiz os dados observados e visa estimar o vetor $\theta$ de parâmetros (ou a hipótese acerca dos parâmetros) que melhor quantifique as chances de que os fatos (dados) se repitam. Desta forma, a estimativa de máxima verossimilhança de $\theta$ é o vetor $\hat{\theta}$ que maximize $L(\theta)$ na expressão (8).

$$
L(\theta)=\prod_{\delta_{i}=1} f\left(t_{i} / \theta\right) \prod_{\delta_{i}=0} R\left(t_{i} / \theta\right)
$$

\section{Onde}

$f\left(t_{i} / \theta\right)$ é a função densidade de probabilidade, indexada pelo vetor $\hat{\theta}$ de parâmetros

$R\left(t_{i} / \theta\right)$ é a função de confiabilidade, indexada pelo vetor $\hat{\theta}$ de parâmetros.

O método de máxima verossimilhança é ainda utilizado para a construção de intervalos de confiança de $95 \%$ para os parâmetros e para as estatísticas de interesse, a exemplo do tempo médio de falhas, mediana, percentis, entre outros. No problema em discussão, este método possibilita estimar estes valores dos parâmetros do modelo de regressão da expressão (1) e suas respectivas variâncias. Estes valores possibilitam a construção de intervalos de confiança 95\% para a previsão de vida discutida, da seguinte forma

$$
L_{i}=\hat{\phi}-1,96 \sqrt{\operatorname{Var}(\hat{\phi})}
$$

$L_{s}=\hat{\phi}+1,96 \sqrt{\operatorname{Var}(\hat{\phi})}$

Onde

$L_{i}$ é o limite inferior

$L_{s}$ é o limite superior

$\hat{\phi}$ é a estimativa de máxima verossimilhança da estatística de interesse

$\operatorname{Var}(\hat{\phi})$ é a sua variância, para as condições do modelo de regressão deste estudo.

Como a função $\hat{\phi}$ de interesse envolve a estimativa de mais de um parâmetro, a obtenção de $\operatorname{Var}(\hat{\phi})$ é feita pela aproximação multivariada do Método Delta (COLOSIMO; GIOLO, 2006), dada pela seguinte equação

$$
\begin{aligned}
& \operatorname{Var}(\hat{\phi})=\operatorname{Var}(\hat{\alpha}) \hat{\phi}^{2}+\operatorname{Var}(\hat{\beta}) x_{0}^{2} \hat{\phi}+\operatorname{Var}(\hat{\sigma}) \hat{\sigma}^{2} \hat{\phi}^{2} \ldots \\
& \ldots+2 \operatorname{Cov}(\hat{\alpha} ; \hat{\beta}) x_{0} \hat{\phi}^{2}+2 \operatorname{Cov}(\hat{\alpha} ; \hat{\sigma}) \hat{\sigma} \hat{\phi}^{2}+2 \operatorname{Cov}(\hat{\beta} ; \hat{\sigma}) \sigma x_{0} \hat{\phi}^{2}
\end{aligned}
$$

Onde

$\hat{\alpha}, \hat{\beta}, \hat{\sigma}$ são as estimativas dos parâmetros do modelo de regressão

$x_{0}$ é o valor da carga utilizada para obter a estatística de interesse, podendo ser uma carga de estresse ou a carga nas condições normais de uso.

Porém, a análise dos dados de falha desta forma não considera as incertezas na variável de estresse. Para que isso seja possível, o modelo de regressão de locação e escala aqui discutido foi tratado no contexto de modelos de regressão com erros de medida, como mostra o item a seguir.

\section{O PROBLEMA DO ERRO DE MEDIDA}

Segundo Carroll et al. (2006), para realização de uma análise de regressão com erros de medida dois requisitos são importantes: a especificação da estrutura dos dados e da estrutura dos erros. A estrutura dos dados é definida pelas propriedades dos valores verdadeiros da variável independente $X_{i}, i=i \ldots n$. Esta variável não pode ser diretamente observada, pois possui erros de medição, por isso, observa-se em seu lugar uma outra variável, denominada neste estudo de $W$. Tradicionalmente, é feita distinção entre o modelo funcional clássico, em que os valores de $X_{i}$ são tratados como uma seqüência de valores constantes fixados, e o modelo estrutural clássico, em que os valores de $X_{i}$ são tratados como variáveis aleatórias.

Ainda de acordo com Carroll et al. (2006), com relação à especificação da estrutura dos erros os modelos podem ser classificados em dois tipos:

- Modelos de Erro, que incluem o modelo de erro de medição clássico;

- Modelos de Calibração, que incluem o modelo de erro de Berkson.

Neste estudo, os verdadeiros valores das variáveis de estresse que não podem ser observados são considerados constantes, o que equivale a dizer que a estrutura dos dados deste estudo é funcional. Com relação à estrutura dos erros, segundo Montenegro (2006), em problemas que utilizam os modelos de locação e escala (equação (26)) em geral, a estrutura do Modelo de Erro é especificada da seguinte forma: 
$W=X+U$

Onde

$U$ é o erro de medida, com média zero e variância $\sigma_{u}^{2}$ $X$ é o verdadeiro valor da variável de estresse, que não pode ser observado diretamente num experimento, devido à existência de erros de medida

$W$ é a variável de estresse observada, que corresponde ao valor verdadeiro de $X$ adicionado de erros aleatórios, dados pela variável $U$.

Assim, quando as incertezas são consideradas na análise, o modelo de regressão de locação e escala, para $Y=\log (T)$, assume a forma da equação 13 .

$$
Y=\mu(X)+\sigma \varepsilon
$$

$$
\begin{aligned}
& \text { Onde } \\
& W=X+U
\end{aligned}
$$

Assim, neste estudo, o modelo dado pela equação (13) passa a ser utilizado como o modelo de regressão para a extrapolação dos dados acelerados para as condições normais de uso. Para gerar a regressão considerando este modelo foi utilizado o método SIMEX, discutido na seqüência.

\section{O MÉTODO SIMEX}

Inicialmente, o método SIMEX foi proposto por Cook e Stefanski (1994) e aprimorado posteriormente por Stefanski e Cook (1995) e por Carroll et al. (1996). O método SIMEX é baseado em simulação e extrapolação e utilizado para estimar e reduzir tendências causadas por erros de medição.

O método SIMEX pode ser entendido em dois passos; simulação e extrapolação. No passo de simulação, erros de medida crescentes e proporcionais a $(1+\lambda) \sigma_{u}^{2}, \lambda \geq$ 0 , são adicionados a variável de estresse original que possibilita verificar a tendência que tais erros provocam nos valores dos parâmetros depois de gerada a regressão. Estes erros são variâncias que correspondem a $\lambda_{m} \sigma_{u}^{2}$ e que somadas aos dados originais geram uma variância total de $\sigma_{u}^{2}+\lambda_{m} \sigma_{u}^{2}=\left(1+\lambda_{m}\right) \sigma_{u}^{2}$ (CARROLL et al., 2006). No passo de extrapolação os valores estimados para os parâmetros em função das incertezas adicionais são modelados apropriadamente e extrapolados para a situação em que os efeitos dos erros são atenuados (LECHNER e POHLMEIER, 2005).

A Figura 1 apresenta uma visão geral do método SIMEX. No passo de simulação, erros com distribuição normal, média zero e a variância dos erros de medida da variável independente $\sigma_{u}^{2}$ são gerados. Em seguida, esses erros são adicionados às variáveis de estresse juntamente com as componentes $\lambda_{m}$, com $0 \leq \lambda \leq 2$. Este procedimento gera novas variáveis aleatórias $W_{(i, b)}\left(\lambda_{m}\right)$ para cada valor de $\lambda_{m}$ fixado. A partir destas novas variáveis e do estimador de máxima verossimilhança são geradas regressões que possibilitam estimar os parâmetros do modelo de regressão de locação e escala, agora considerando as incertezas nas variáveis de estresse. Esta seqüência de procedimentos é repetida B vezes, gerando B valores de cada parâmetro do modelo para cada valor de $\lambda_{m}$ fixado. A estimativa final dos parâmetros modelo é obtida a partir da média dos B valores de cada $\lambda_{m}$, da seguinte forma:

$$
\hat{\beta}_{j}\left(\lambda_{m}\right)=\frac{1}{b} \sum_{1}^{b} \hat{\beta}_{j, b}\left(\lambda_{m}\right)
$$

Onde

$\hat{B}_{j}\left(\lambda_{m}\right)$ é a estimativa SIMEX do parâmetro do modelo de regressão de locação e escala, obtido em função de $\lambda_{m}$

$\lambda_{m}$ é o componente usada para adicionar erros crescentes na variável de estresse do modelo de regressão, onde $0 \leq$ $\lambda \leq 2$

$\hat{B}_{i, j}\left(\lambda_{m}\right)$ são os valores estimados para os parâmetros do modelo de regressão, no passo de simulação do SIMEX, obtidos em função de $\lambda_{m}$.

Este procedimento permite obter os pares $\left(\lambda, \beta_{j}\left(\lambda_{m}\right)\right)$, com os quais são construídos gráficos de tendência dos parâmetros para cada valor de $\lambda_{m}$ (Figura 1), que são extrapolados para a condição em que os efeitos das incertezas são considerados na análise.

De acordo com Cook e Stefanski (1994), quando $\lambda=-1$ obtém-se o valor verdadeiro da variável independente $X_{i}$ com as incertezas experimentais influenciando o experimento real e a análise. As estimativas obtidas neste caso são denominadas de estimativas SIMEX. Quando $\lambda=0$, tem-se de fato a variável experimental influenciada por incertezas, ou seja, a variável $W$, mas a análise as desconsidera. As estimativas obtidas nesta condição são chamadas de estimativas convencionais.

\section{RESULTADOS E DISCUSSÕES}

O conjunto de dados reais utilizado foi coletado por Sasseron (2005) e corresponde a um ensaio acelerado de relés eletromagnéticos. Um relé tem a função de ligar e desligar motores, resistências, entre outros equipamentos. O tipo analisado no ensaio aqui discutido é composto por uma bobina enrolada em um núcleo fixo de material magnético que, ao ser submetido à corrente elétrica, produz um campo magnético que gera uma força eletromecânica responsável pelo procedimento de liga-desliga do relé. O modo de falha 
considerado foi a falha no processo liga-desliga, causada pelo aumento da corrente de carga que passa nos contatos. A Tabela 1 apresenta as especificações do relé de acordo com o fabricante. Estas especificações foram utilizadas como informações importantes para o planejamento do ensaio e como parâmetros para discussão do resultado final de ambas as análises, convencional e SIMEX.

A determinação dos valores das cargas de estresse considerou os limites de projeto do relé, além dos cálculos das incertezas das resistências equivalentes $R_{e q}$ com base no INMETRO (2003). Isso resultou na definição experimental final dos valores de $6.12 \mathrm{~A}, 9.25 \mathrm{~A}, 11.6 \mathrm{~A}$ e $15.27 \mathrm{~A}$ para a variável de estresse, conforme mostrado na Tabela 2, com as respectivas incertezas combinadas $u c(I)$ também mostradas na tabela.

Para a realização do ensaio, foram consideradas 4 amostras aleatórias com 16 relés para cada uma. Os tempos de falha ou censura obtidos são apresentados na Tabela 3. Como variável indicadora de censura utilizou-se $\delta=1$ para dados completos e $\delta=0$ para dados censurados. A coluna da esquerda indica a ordem crescente dos tempos de falhas. As colunas denominadas "ciclos" mostram os números de

Figura 1: Método SIMEX, passos de Simulação e Extrapolação.

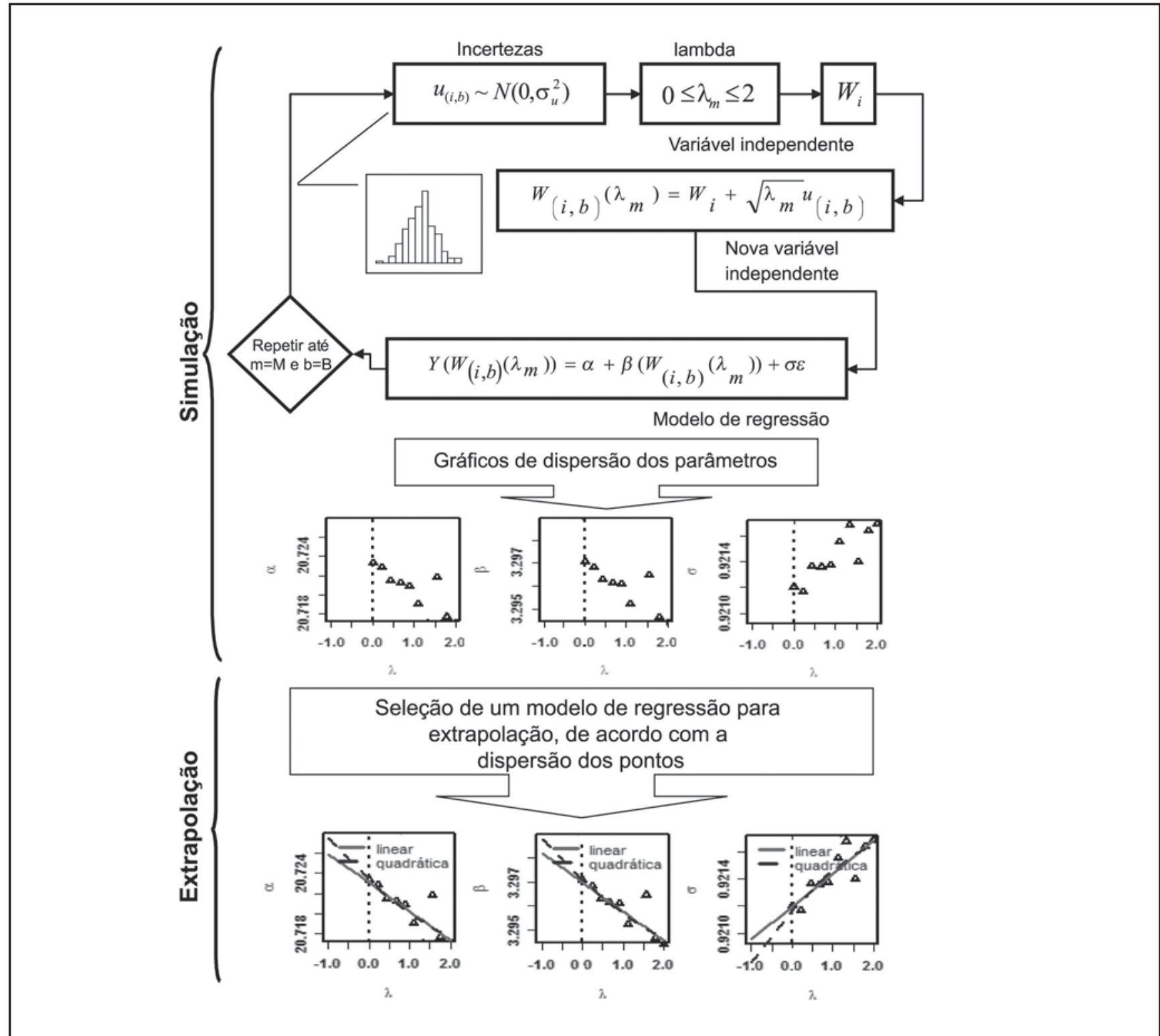


ciclos liga-desliga executados por cada relé até ter sido retirado do teste por falha ou por censura.

As análises convencional e SIMEX foram realizadas para obtenção dos tempos de falha para as condições normais de uso de $5 \mathrm{~A}$, de acordo com as especificações do fabricante do relé (Tabela 1). Para isso foi ajustado o modelo de regressão Potência Inversa-log normal (PAPA, 2007).

Neste estudo foram obtidas as estimativas $B_{10}$, que representam o tempo em que $90 \%$ dos relés não falharam; $B_{50}$ ou mediana, que representa o tempo para que $50 \%$ dos relés ainda sobrevivam e MTTF (Mean Time Between Failures), que representa o tempo médio de falha dos relés.

A discussão final dos resultados deste estudo é feita considerando as estimativas de $B_{50}$. Isso pelo fato de que, segundo Sterl (1997), ela é utilizada para estimar a vida elétrica característica do relé, em especial para a estimativa de seu tempo de garantia. Assume-se a partir de Sterl (1997) que a e vida típica seja obtida de duas formas: a primeira, que considera a estimativa do limite inferior do intervalo de $95 \%$ de confiança obtido para o $B_{50}$, e a segunda, que utiliza o menor valor obtido para um grande número desta estimativa.

Primeiramente foram obtidas as estimativas da análise convencional, que não considera na análise as incertezas nas variáveis de estresse. Apesar deste resultado não ser o mais importante neste estudo, ele serve de base para comparação das estimativas obtidas com a implementação do SIMEX. A partir desta comparação, verifica-se o efeito das incertezas na variável de estresse sobre estas estimativas de tempos de falha.

A análise SIMEX utilizou os dados da Tabela 3, o modelo de regressão Potencia Inversa - log-normal (equação 1), o estimador de máxima verossimilhança (equação 9) e as incertezas da variável de estresse dadas pelas incertezas combinadas da Tabela 2 , representadas por $u_{c}(I)$, que correspondem ao desvio padrão das incertezas da variável de estresse. No passo de simulação foram realizadas 1000 ite-

Tabela 1: Características do relé segundo o fabricante.

\begin{tabular}{|l|c|}
\hline \multicolumn{1}{|c|}{ CARAGTERÍSTICAS DO RELÉ } & DEFINIÇÃO DO FABRIGANTE \\
\hline Tensão nominal & $24 \mathrm{VCC}$ \\
\hline Corrente nominal da bobina & $21 \mathrm{~mA}$ \\
\hline Consumo do relé & $0,5 \mathrm{~W}$ \\
\hline Corrente nominal (resistiva) & $5 \mathrm{~A}$ \\
\hline Corrente nominal (indutiva) & $2 \mathrm{~A}$ \\
\hline Temperatura máxima de funcionamento & $55^{\circ} \mathrm{C}$ \\
\hline Tempo mínimo esperado de vida $\left(B_{50}\right)$ & 1000000 de ciclos \\
\hline Tempo limite de chaveamento & $30 \mathrm{~ms}$ \\
\hline Tempo mínimo entre chaveamentos & $2 \mathrm{~s}$ \\
\hline
\end{tabular}

Fonte: Abackerli et al. (2006)

Tabela 2: Definição experimental da carga de estresse.

\begin{tabular}{|c|c|c|c|c|c|c|}
\hline ESTRESSE NOMINAL** & $\boldsymbol{R}_{\mathrm{EQ}}^{*}$ & $u_{c}\left(R_{E Q}\right)^{*}$ & $r^{* *}$ & $u_{c}(I)^{* * *}$ & $U(I)^{* * * *}$ & $U(1)[\%]$ \\
\hline 6 & 3,922 & 0,010 & 6,12 & 0,034 & 0,068 & 1,1 \\
\hline 9 & 2,600 & 0,012 & 9,22 & 0,063 & 0,126 & 1,4 \\
\hline 12 & 2,073 & 0,012 & 11,60 & 0,088 & 0,176 & 1,5 \\
\hline 15 & 1,572 & 0,009 & 15,27 & 0,116 & 0,232 & 1,5 \\
\hline
\end{tabular}

Fonte: Abackerli et al. (2006) 
rações e no passo de extrapolação utilizaram-se os modelos linear e quadrático. Os resultados gráficos da análise SIMEX são verificados na Figura 2.

Observa-se na Figura 2 que as estimativas dos parâmetros de escala $\sigma$ crescem com o aumento das incertezas, em função de $\lambda$. Este comportamento de $\sigma$ era esperado, uma vez que este aumento tende a fornecer estimativas menos precisas (CARROL et al., 2006). Já com relação ao comportamento dos parâmetros $\alpha$ e $\beta$, tinha-se apenas a expectativa de que o aumento das incertezas em função de $\lambda$ influenciaria de alguma forma suas estimativas. Ainda na Figura 2, as curvas representadas pela linha contínua (regressão linear) e linha tracejada (regressão quadrática) mostram a extrapolação dos valores dos parâmetros para a condição em que $\lambda=-1$. Este procedimento possibilita conhecer as estimativas SIMEX dos parâmetros do modelo de regressão de locação e escala e seus respectivos intervalos de $95 \%$ de confiança, mostrados na Tabela 4 .

Nota-se que ambos os modelos linear e quadrático apresentam estimativas muito próximas, tanto para os valores do parâmetro quanto para o desvio padrão, levando à conclusão de que, neste caso, as duas extrapolações geram resultados satisfatórios. Para apresentação final dos resultados optou-se pelas estimativas do modelo quadrático, pois esta regressão apresentou o valor de $R^{2}$ ajustado maior em relação ao linear, indicando que o modelo quadrático possibilita melhor ajuste. A Tabela 5 apresenta as estimativas dos parâmetros para as análises convencional e SIMEX com seus respectivos desvio padrão.

A partir das estimativas dos parâmetros, apresentadas na Tabela 5 foram obtidas as estimativas dos tempos de falha para a condição usual de $5 \mathrm{~A}$ e seus respectivos intervalos de $95 \%$ de confiança. Estas estimativas são verificadas na Tabela 6.

Com relação ao efeito das incertezas na variável de estresse sobre as estimativas dos tempos de falha, verifica-se primeiramente que os resultados calculados mostram variações de apenas $0,2 \%$ para $\mathrm{B}_{10}, 0,1 \%$ para $\mathrm{B}_{50}$ e $0,1 \%$ para o MTTF. Assim, do ponto de vista prático das estimativas dos tempos de falha para a condição de $5 \mathrm{~A}$, pode-se dizer que

Tabela 3: Dados experimentais.

\begin{tabular}{|c|c|c|c|c|c|c|c|c|}
\hline \multirow{3}{*}{$\mathbf{n}$} & \multicolumn{8}{|c|}{ NÍVEL DE ESTRESSE } \\
\hline & \multicolumn{2}{|c|}{$6.12 \mathrm{~A}$} & \multicolumn{2}{|c|}{9.25 A } & \multicolumn{2}{|c|}{$11.6 \mathrm{~A}$} & \multicolumn{2}{|c|}{$15.27 A$} \\
\hline & GIGLOS & $\delta$ & GICLOS & $\delta$ & CICLOS & $\delta$ & CIGLOS & $\delta$ \\
\hline 1 & 480406 & 1 & 73352 & 1 & 31085 & 1 & 45588 & 1 \\
\hline 2 & 551402 & 1 & 98033 & 1 & 98888 & 1 & 111632 & 1 \\
\hline 3 & 813123 & 1 & 236320 & 1 & 109381 & 1 & 113205 & 1 \\
\hline 4 & 1868621 & 0 & 264699 & 1 & 131948 & 1 & 132499 & 1 \\
\hline 5 & 1868621 & 0 & 420441 & 1 & 132251 & 1 & 153180 & 1 \\
\hline 6 & 1868621 & 0 & 455503 & 1 & 136459 & 1 & 163699 & 1 \\
\hline 7 & 1868621 & 0 & 495202 & 1 & 13840 & 1 & 164788 & 1 \\
\hline 8 & 1868621 & 0 & 550119 & 1 & 143818 & 1 & 179237 & 1 \\
\hline 9 & 1868621 & 0 & 733991 & 1 & 191223 & 1 & 204592 & 1 \\
\hline 10 & 1868621 & 0 & 894067 & 1 & 208244 & 1 & 211918 & 1 \\
\hline 11 & 1868621 & 0 & 953393 & 1 & 209839 & 1 & 216590 & 1 \\
\hline 12 & 1868621 & 0 & 1793409 & 1 & 229059 & 1 & 251962 & 1 \\
\hline 13 & 1868621 & 0 & 1817479 & 1 & 254377 & 1 & 266807 & 1 \\
\hline 14 & 1868621 & 0 & 1882756 & 1 & 433524 & 1 & 300019 & 1 \\
\hline 15 & 1868621 & 0 & 2064540 & 1 & 500092 & 1 & 367829 & 1 \\
\hline 16 & 1868621 & 0 & 3309823 & 0 & 522573 & 1 & 526826 & 1 \\
\hline
\end{tabular}

Fonte: Sasseron (2005) 
nas condições de teste de Sasseron (2005) com incertezas relativamente pequenas na variável de estresse os seus efeitos são praticamente nulos. A confirmação de que as incertezas sejam os fatores determinantes dessas pequenas diferenças pode ser feita também por simulação, onde seja avaliado tanto o vício quanto a precisão das estimativas.

Figura 2: Resultado gráfico da análise SIMEX.


Tabela 4: Estimativas SIMEX dos parâmetros com extrapolação linear e quadrática.

\begin{tabular}{|c|c|c|c|c|}
\hline & PARÂMETROS & $\alpha_{\text {SIMEX }}$ & $\beta_{\text {SIMEX }}$ & $\sigma_{\text {SIMEX }}$ \\
\hline \multirow{4}{*}{ Linear } & Valor & 20,726 & 3,198 & 0,921 \\
\hline & Desvio padrão & $5,62 e^{-04}$ & $2,48 e^{-04}$ & $0,36 e^{-05}$ \\
\hline & Li p/ 95\% confiança & 20,724 & 3,198 & 0,921 \\
\hline & Ls p/ 95\% de confiança & 20,728 & 3,299 & 0,921 \\
\hline \multirow{4}{*}{ Quadrático } & Valor & 20,728 & 3,199 & 0,921 \\
\hline & Desvio Padrão & $7,94 e^{-04}$ & $3,451 e^{-04}$ & $5,122 \mathrm{e}^{-05}$ \\
\hline & Li p/95\% confiança & 20,720 & 3,296 & 0,920 \\
\hline & Ls p/95\% de confiança & 20,726 & 3,304 & 0,921 \\
\hline
\end{tabular}

Tabela 5: Estimativas SIMEX e convencional dos parâmetros com os seus respectivos desvios padrão.

\begin{tabular}{|l|l|l|l|}
\hline \multicolumn{1}{|c|}{ PARÂMETROS } & \multicolumn{1}{|c|}{$\alpha$ (DESVIO PADRÃO) } & $\beta$ (DESVIO PADRÃO) & \multicolumn{1}{c|}{$\sigma$ (DESVIO PADR ÃO) } \\
\hline Estimativa convencional & $20,723(0,9506)$ & $3,297(0,3990)$ & $0,921(0,1026)$ \\
\hline Estimativa SIMEX & $20,728(0,9510)$ & $3,299(0,3992)$ & $0,920(0,1028)$ \\
\hline
\end{tabular}


Além disso, tomando o limite inferior de $95 \%$ de confiança para $\mathrm{B}_{50}$ nas estimativas convencional e SIMEX, vê-se uma redução em torno de 550000 ciclos na vida característica do relé quando as incertezas são consideradas na análise. Neste caso, para os dados e as condições de teste deste estudo, o valor estimado considerando as incertezas é bem maior que o valor dado pelo fabricante na Tabela 1 , mesmo considerando as incertezas. Conforme já discutido, este resultado tem relevância prática no desenvolvimento de produtos assumindo a hipótese de Sterl (1997), em que a vida mínima do relé (Tabela 1) seja determinada pelo fabricante a partir do limite inferior deste intervalo (vide Li para $\mathrm{B}_{50}$, Tabela 6). Portanto, sob este enfoque, percebese uma provável superestimativa de cerca de $28 \%$ na vida típica do relé quando incertezas mesmo pequenas não são consideradas na análise.

Ainda, considerando os resultados da Tabela 6 poderse-ia concluir que a análise convencional apresenta melhor resultado que a análise SIMEX devido ao seu menor desvio padrão. Porém, é importante que sejam avaliadas as estimativas pontuais e seus respectivos intervalos de confiança de $95 \%$, pois os resultados aqui obtidos indicam que provavelmente o efeito da incerteza se manifeste mais fortemente na precisão das estimativas (dispersão dos resultados) do que no seu vício (valores das estimativas). Como discutido, este cuidado deve ser tomado principalmente para os casos em que a dispersão dos resultados seja usada pelo fabricante do produto para a determinação da vida (Tabela 1).

$\mathrm{Na}$ prática diária das empresas fica evidente que a definição experimental da variável de estresse depende da qualidade e da exatidão do instrumento de medição utiliza- do. Por isso, para verificar o efeito de diferentes valores de incerteza, optou-se por implementar o SIMEX com valores de incertezas variando desde $0 \%$ (valor moderado) a $8 \%$ (valor severo). Estes valores de incerteza correspondem a porcentagens em relação ao valor nominal do estresse utilizado por Sasseron (2005). A Tabela 7 mostra estes valores em ampères.

Para esta verificação do efeito dos diferentes valores de incertezas, o método SIMEX foi usado da mesma forma que para os dados e incertezas de Sasseron (2005), porém considerando-se as incertezas da Tabela 7. Como resultados obtiveram-se as estimativas de tempos de falha mostrados na Tabela 8 .

Verifica-se na Tabela 8 que, do ponto de vista de análise das incertezas, pode-se dizer que, para as cargas de estresse com incertezas combinadas a partir de $4 \%$, os efeitos nas estimativas começam a ser importantes e devem ser considerados nas estimativas de confiabilidade e tempos de garantia. Estes resultados podem ser graficamente verificados na Figura 3.

Verifica-se na Figura 3 que as estimativas dos tempos de falha tendem a aumentar para incertezas superiores a $3 \%$. Este aumento é mais acentuado principalmente para as estimativas do MTTF, porém, também verificadas para $B_{50}$ e $B_{10}$.

Os resultados apresentados na Tabela 8 correspondem às estimativas dos tempos de falha. Se forem consideradas apenas estas estimativas pontuais isoladamente poder-se-ia dizer que os efeitos das incertezas não são importantes nas estimativas do tempo de garantia do relé, especialmente para os dados de Sasseron (2005), cujas incertezas são relativamente pequenas. Porém, as estimativas dos intervalos de

Tabela 6: Estimativas dos tempos de falha do relé para as condições normais de uso.

\begin{tabular}{|c|c|c|c|}
\hline ESTIMATIVAS DE TEMPO DE FALHA * & CONVENGIONAL & SIMEX & EFEITOS (\%) \\
\hline B $_{\mathbf{1 0}}$ & $\mathbf{1 5 2 3 2 2 1}$ & $\mathbf{1 5 2 5 8 4 9}$ & $\mathbf{0 , 2}$ \\
\hline $\mathrm{Li}$ & 614678 & 444453 & $-28,0$ \\
$\mathrm{LS}$ & 2431764 & 2607245 & $\mathbf{7 , 0}$ \\
\hline $\mathbf{B}_{\mathbf{5 0}}$ & $\mathbf{4 9 5 9 9 5 4}$ & $\mathbf{4 9 6 6 7 2 6}$ & $\mathbf{0 , 1}$ \\
\hline $\mathrm{Li}$ & 2001531 & 1446721 & $-28,0$ \\
\hline Ls & 7918377 & 8486731 & $\mathbf{7 , 0}$ \\
\hline MTF & $\mathbf{7 5 8 1 4 4 5}$ & $\mathbf{7 5 8 9 8 3 6}$ & $\mathbf{0 , 1}$ \\
\hline Li & 3059403 & 2210788 & $-27,0$ \\
\hline Ls & 12103488 & 12968885 & 7,0 \\
\hline
\end{tabular}


confiança de $95 \%$ indicam para o fato de que, apesar de as estimativas pontuais indicarem efeitos aparentemente pouco expressivos, tais incertezas podem ser significativas quando estes intervalos são considerados. Por isso, vale ressaltar que os efeitos das incertezas nas variáveis de estresse podem gerar estimativas de tempos de garantia que não correspondem à realidade do produto ensaiado. Esse é um resultado preponderante, tendo em vista a qualidade entre produtos concorrentes. Nesse sentido, é importante enfatizar a utilidade desse tipo de ensaio ao longo do processo de desenvolvimentos, de novos produtos, visando a obtenção de produtos mais robustos, em termos de confiabilidade. Assim como os diversos outros tipos de estudos de confiabilidade, o ALT pode ser utilizado nos diversos estágios de desenvolvimento de um novo produto, notadamente na fase de projeto detalhado, onde é necessária a homologação do produto em desenvolvimento. No entanto, não existe um impedimento de que este ensaio seja usado nas fases iniciais de desenvolvimento, mesmo na definição do conceito do produto, a partir de alternativas existentes. Quanto aos tipos de produtos, projetos de melhoria, derivativos (também chamados de variantes) ou produtos inovadores, o método proposto pode ser aplicado, principalmente nos dois primeiros casos, em que geralmente têm-se dados de garantia disponíveis de produtos similares.

Tabela 7: Incertezas Combinadas $\mathrm{u}_{\mathrm{c}}$.

\begin{tabular}{|c|c|c|c|c|}
\hline \multirow{2}{*}{ INCERTEZAS (\%)* } & \multicolumn{3}{|c|}{ GARGAS DE ESTRESSE (A) } \\
\cline { 2 - 5 } & $\mathbf{6 , 1 2}$ & $\mathbf{9 , 2 5}$ & $\mathbf{1 1 , 6 0}$ & $\mathbf{1 5 , 2 7}$ \\
\hline 1 & 0,061 & 0,093 & 0,116 & 0,153 \\
\hline 2 & 0,122 & 0,185 & 0,232 & 0,305 \\
\hline 3 & 0,184 & 0,278 & 0,348 & 0,458 \\
\hline 4 & 0,250 & 0,365 & 0,464 & 0,611 \\
\hline 5 & 0,313 & 0,456 & 0,580 & 0,764 \\
\hline 6 & 0,367 & 0,555 & 0,696 & 0,916 \\
\hline 7 & 0,438 & 0,638 & 0,812 & 1,069 \\
\hline 8 & 0,500 & 0,720 & 0,928 & 1,221 \\
\hline *Incertezas combinadas $u_{c}$ dados em Ampères (A). & & & \\
\hline
\end{tabular}

Tabela 8: Resultado das estimativas de tempos de falha para diferentes valores de incertezas.

\begin{tabular}{|c|c|c|c|c|c|c|}
\hline \multirow{2}{*}{ INCERTEZAS (\%) } & \multicolumn{6}{|c|}{ TEMPOS DE FALHA ESTIMADOS SIMEX } \\
\cline { 2 - 8 } & $\mathbf{B}_{10}$ & EFEITOS (\%) & $\mathbf{B}_{50}$ & EFEITOS (\%) & MTFF & EFEITOS (\%) \\
\hline 0 & 1523221 & $5,33 e^{-12}$ & 4959953 & $5,33 e^{-12}$ & 7581445 & $5,33 e^{-12}$ \\
\hline 1 & 1526902 & 0,24 & 4967652 & 0,15 & 7588507 & 0,09 \\
\hline 2 & 1535739 & 0,80 & 4984884 & 0,50 & 7602229 & 0,30 \\
\hline 3 & 1552383 & 1,90 & 5028480 & 1,40 & 7657342 & 1,00 \\
\hline 4 & 1592502 & 4,00 & 5112723 & 3,10 & 7761497 & 2,40 \\
\hline 5 & 1642443 & 6,90 & 5220677 & 5,30 & 7894740 & 4,10 \\
\hline 6 & 1694711 & 8,10 & 5225718 & 6,00 & 7919555 & 4,50 \\
\hline 7 & 1739347 & 13,8 & 5430702 & 9,60 & 8127201 & 7,20 \\
\hline 8 & 1830629 & 17,0 & 5590507 & 12,70 & 8315653 & 9,70 \\
\hline
\end{tabular}


No caso de produtos inovadores, conforme o nível de ruptura tecnológica, essa utilização fica mais difícil, uma vez que a disponibilidade de dados é limitada. Não obstantemente, a adoção do ALT em conjunto com a análise de incertezas vem a contribuir com o desenvolvimento de produtos eficazes sob o ponto de vista da probabilidade de falhas.

\section{CONCLUSÕES}

O estudo para verificar o efeito das incertezas na variável de estresse em ensaios acelerados foi feito utilizando um conjunto de dados reais. O produto testado neste ensaio foi um relé eletromagnético, para o qual se considerou um modo de falha. Utilizou-se o modelo de regressão Potência Inversa - log-normal e o método usado para investigar estes efeitos foi o método SIMEX.

Os resultados indicam que para as cargas de estresse com incertezas maiores que $4 \%$ do seu valor nominal, o efeito das incertezas foi importante tanto sobre as estimativas pontuais dos percentis dos tempos de falha como sobre os limites inferiores e superiores de intervalo de $95 \%$ de confiança. Porém, para incertezas menores a $4 \%$, os efeitos das incertezas nas estimativas pontuais dos percentis de interesse podem ser desconsiderados na análise, mas, ainda, para incertezas muito pequenas, os intervalos de confiança de 95\% são superestimados. Porém, conclusões mais decisivas sobre as estimativas da vida e da garantia dos relés obtidas

Figura 3: Estimativas de tempos de falha para diferentes valores de incertezas

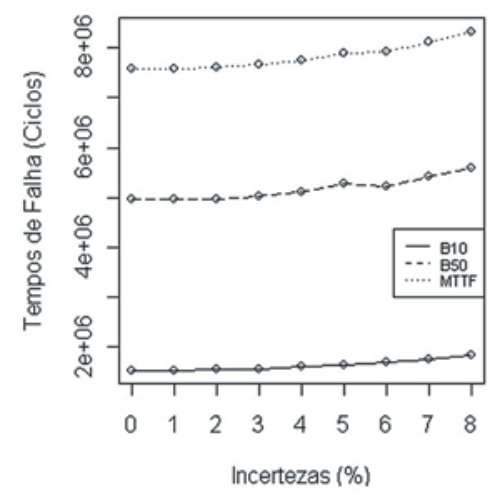

Artigo recebido em 21/06/2007

Aprovado para publicação em 26/11/2007 a partir dos intervalos de confiança de $95 \%$ necessitam de outros estudos via simulação que permitam obter os vícios das estimativas convencional e SIMEX. Tais estudos serão objetos de futura publicação.

No que se refere ao problema de engenharia em discussão, as estimativas de tempos de falha obtidos da implementação do método SIMEX foram similares às obtidas na análise convencional. Porém, a análise SIMEX proporcionou limites inferiores de $95 \%$ de confiança cerca de 30\% menores que os obtidos na análise convencional, mostrando que as incertezas podem afetar tanto a dispersão quando o valor da estimativa. Desta forma, considerando a vida típica do relé dada em função do limite inferior de confiança, pode-se dizer que as influências na definição das cargas de estresse sejam importantes mesmo para incertezas menores que $4 \%$. Porém, a confirmação deste resultado depende de estudos de simulação para obtenção dos vícios na estimativa convencional e SIMEX.

Segundo o observado no presente ensaio e, hipoteticamente, assumindo que o relé testado seja utilizado em um equipamento que realiza uma média de 500 ciclos ligadesliga ao dia, sua vida estimada seria de aproximadamente 5 anos para atingir os 1.000 .000 de ciclos informados pelo fabricante. Entretanto, se a vida mínima é determinada pelo fabricante a partir do limite inferior de confiança, sem considerar incertezas, sua vida típica estaria superestimada em $28 \%$ se a incertezas discutidas, mesmo pequenas, estivessem presentes no ensaio acelerado do produto. Em termos práticos, isso implicaria na necessidade de reduzir a vida estimada para cerca de 4 anos, o que não seria feito se as incertezas fossem negligenciadas na análise dos resultados do ensaio acelerado.

Do ponto de vista da importância do resultado para o desenvolvimento de um novo produto, pode-se dizer que os efeitos destas incertezas não acarretariam atrasos no lançamento do produto. No entanto, poderia vir a incorrer em prejuízos financeiros, pois não considerar as incertezas na análise pode significar estimativas de tempos de falha que não correspondem à realidade. Além disso, a utilização deste tipo de ensaio resulta em produtos mais robustos, sob o ponto de vista da confiabilidade, sejam estes melhorias de produtos existentes, derivativos ou, embora com algumas limitações, produtos inovadores, devido às características da existência de dados mais escassos desse último tipo. Nota-se, portanto, que os ensaios acelerados, bem como a proposta do presente estudo, vêm a contribuir com o desenvolvimento de novos produtos. 


\section{- Referências}

ABACKERLI, A.; SASSERON, P. L.; DEMOLIN, F. L. A Test JIG for Relay's Accelerated Testing. In: $5^{\text {th }}$ INTERNATIONAL CONFERENCE ON MECHANICS AND MATERIALS IN DESIGN, Porto, Jul. 2006.

AHMED, J. U. Modern Approaches to Product Reliability Improvement. International Journal of Quality \& Reliability Management, Bradford, v. 13, n. 3, p.2741, Apr. 1996.

AW, K. C. Integrating Quality and Reliability Assessment into Product Development Process: A New Zealand Case Study. International Journal of Quality $\&$ Reliability Management, Bradforf, v. 22, n. 5, p. 518-530, Jun. 2005

BOOKER, J. D. Industrial Practice in Designing for Quality. International Journal of Quality \& Reliability Management, Bradford, v. 20, n. 3, p. 288-302, Apr. 2003.

CARROLL, J. R. et al. Measurement Error in Nonlinear Models: A Modern Perspective. New York: Chapman \& Hall, Monographs on statistics and Applied Probability 63, 2. ed., 2006, 456 p.

COlOSIMO, E.; GIOLO, S. R. Análise de sobrevivência Aplicada. 1. ed., São Paulo: Edgard Blucher, 2006. 367 p.
COOK, J.; STEFANSKI, L. A. A Simulation Extrapolation Method in Parametric Measurement Error Models. Journal of the American Statistical Association, Alexandria, v. 98, n. 428, p. 1314-1328, Dec.1994.

CORDEIRO, G. Introdução à Teoria de Verossimilhança. In: SIMPÓSIO NACIONAL DE PROBABILIDADE E ESTATÍSTICA, Rio de Janeiro, 1992, 10p.

ELLEKLAER, M. R.; BISGARD, S. The Use of Experimental Design in the Development of New Product. International Journal of Quality Science, Fargo, v. 3, n. 3, p. 254274, Sep. 1998.

FREITAS, M. A.; COLOSIMO, E Confiabilidade: Análise de Tempos de Falh e Testes de Vida Acelerados. 1. ed. Belo Horizonte: Fundacão Christiano Ottoni, 1997. 326 p. (Série Ferramentas da Qualidade).

INMETRO. Guia para a Expressão da Incerteza da Medição, 3. ed. Brasileira em Língua Portuguesa. Rio de Janeiro, 2003. $120 \mathrm{p}$

KAHN, K. B.; BARCZAK, G.; MOSS, R. Dialogue on Best Practices in New Product Development Perspective Establishing an NPD Best Practices Framework. Journal of Product Innovation
Management, Philadelphia, v. 23, p. 106116, Mar. 2006.

LECHNER, S.; POHLMEIER, W. Data Masking by Noise Addition and the Estimation of Nonparametric Regression Models. Jahrbucher Fur Nationalokonomie and Statistik, Sttutgard, v. 5, n. 225, p. 517-528, Sep. 2005.

MACKANE, S. W: ESCOBAR, L.A.:MEEKER, W. Q. Sample Size and Number of Failure Requirements for Demonstration Tests with Log-Location-Scale Distributions and Failure Censoring. American Statistical Association and the American Society for Quality, New Orleans, v. 47, n. 2, p. 182190, Mar. 2005.

MONTENEGRO, L. C. C. Modelo de Efeito Aleatório e Erros de Medida. 2006. 196 f. Tese - Curso de Ciências, Departamento de Instituto de Matemática e Estatística, USP, São Paulo, 2006.

NELSON, W. Accelerated Testing: Statistical Models, Test Plans and Data Analysis. Second Edition, New Jersey: John Wiley and Sons, 2004. $601 \mathrm{p}$

PAPA, M. C. O. Análise do Efeito das Incertezas nas Variáveis de Estresse em Ensaios Acelerados, Dissertação - Programa de Pós-Graduação em Engenharia de Produção, Universidade Metodista de
Piracicaba - Unimep, Santa Bárbara D'Oeste, 2007.

R Development Core Team (2007), $R$ : A language and environment for statistical computing. R Foundation for Statistical Computing, Vienna, Austria ISBN 3-900051-07-0, URL http://www.rproject.org.

SASSERON, P. L. Estudo Experimental de Ensaio Acelerado Aplicado a Relés, 2005, 104 p., Dissertação - Programa de PósGraduação em Engenharia de Produção, Universidade Metodista de Piracicaba Unimep, Santa Bárbara D’Oeste, 2005.

STEFANSKI, L. A.; COOK, J. Simulation Extrapolation: the Measurement Errors ackknife. Journal of the American Statistical Association, Alexandria, v. 90, n. 432, p. 1247-1256, Dec. 1995.

STERL, N. Power Relays. $1^{\text {th }}$ ed., Vienna: Eh-schrack Components AG, 1997, $141 \mathrm{p}$.

THEIJE S. M.; SANDER, P. C.; BROMBACHER, A. C. Reliability tests to Control Design Quality: a Case Study. International Journal of Quality \& Reliability Management, Bradford, v. 15, n. 6, p. 599618, Sep. 1998.

\section{- Agradecimentos}

Os autores agradecem as recomendações e sugestões dos revisores que, sem dúvida, aprimoraram a versão final do artigo.

\section{- Sobre os autores}

\section{Maria Célia de Oliveira Papa}

Núcleo de Gestão da Qualidade e Metrologia - NGQM

Faculdade de Engenharia, Arquitetura e Urbanismo - FEAU

Universidade Metodista de Piracicaba - UNIMEP

End.: Rod. 306, km 1 - CEP 13450-000 - Santa Bárbara D’Oeste - SP - Brasil

E-mail: macpapa@unimep.br; mceliamat@yahoo.com.br

Alvaro J. Abackerli

Núcleo de Gestão da Qualidade e Metrologia - NGQM

Faculdade de Engenharia, Arquitetura e Urbanismo - FEAU

Universidade Metodista de Piracicaba - UNIMEP

End.: Rod. Santa Bárbara - Iracemápolis, km 1 - CEP 13450-000 - Santa Bárbara d'Oeste - SP - Brasil

E-mail: abakerli@unimep.br

\section{Paulo Augusto Cauchick Miguel}

Núcleo de Gestão da Qualidade e Metrologia - NGQM

Faculdade de Engenharia, Arquitetura e Urbanismo - FEAU

Universidade Metodista de Piracicaba - UNIMEP

End.: Rod. SP 306, km 1 - CEP 13450-000 - Santa Bárbara d'Oeste - SP - Brasil

E-mail: paulo.miguel@poli.usp.br 